\title{
JOANNA FIRAZA
}

\section{Unter der Glasglocke: generationelle Befindlich- keiten in Zoë Jennys Das Blütenstaubzimmer}

\begin{abstract}
Debiutancka powieść Zoë Jenny Kwietny pyt (1997, wydanie polskie w tłumaczeniu Reginy Tyszkiewicz, 1999) wpisuje się z jednej strony w tendencję literacką ,Fräuleinwunder', z drugiej w tradycję powieści adolescencyjnej. Na uznanie zasługuje plastyczny, zmysłowy język powieści, w którym wyraża się zwrócona do wewnątrz, pierwszoosobowa perspektywa narracyjna. Podjęta tu próba szczegółowej analizy poetyki tekstu szwajcarskiej autorki wykazała zaskakujace pokrewieństwo do autobiograficznej powieści Sylvii Plath Szklany klosz (1963). Intertekstualne podejście rzuca światło na latentną intencję tekstu wyjścia poza horyzont indywidualny czy narodowy oraz na uniwersalny przekaz na temat współczesnej kondycji jednostki, który nadaje tekstowi wydźwięk tragiczny.
\end{abstract}

Zoë Jennys Debütroman Das Blütenstaubzimmer (1997) schreibt sich einerseits in die literarische Tendenz des sogenannten ,Fräuleinwunders', andererseits in die Tradition des Adoleszenzromans ein. Als Leistung des Textes ist seine plastische, sinnliche Sprache anzusehen, in der sich die nach innen gekehrte Perspektive der Ich-Erzählerin ausdrückt. Der hier vorgenommene Versuch einer differenzierten Analyse der Poetik des Textes ergibt eine verblüffende Nähe zu Sylvia Plaths autobiographischem Roman Die Glasglocke (1963). Der intertextuelle Ansatz wirft ein Licht auf den latenten Anspruch des Textes, über das Individuelle und Nationale hinauszugehen, sowie auf die universelle Botschaft über gegenwärtige Befindlichkeiten, die dem Text einen tragischen Unterton verleiht.

Das Blütenstaubzimmer, a debut novel by Zoë Jenny published in 1997 (The Pollen Room, 1999, English translation by Elizabeth Gaffney), can be perceived as a book belonging to the ,Fräuleinwunder' and adolescent novel traditions. What should be exceptionally appreciated is the artistic and sensuous language that reflects a looking inward first person narrative perspective. The thorough analysis of the poetics used by the Swiss author surprisingly bears resemblance to The Bell Jar, an autobiographical 
novel by Sylvia Plath (1963). An inter-textual approach sheds light on the latent intention of the text to go beyond an individual or national level and on the universal message on a contemporary condition of man and its tragic overtones.

Der Debütroman der Schweizer Autorin Zoë Jenny Das Blütenstaubzimmer (1997) ${ }^{1}$ gehört zu den Texten der deutschsprachigen Literatur aus den späten 1990er Jahren, die das literarische Fräuleinwunder mit konstituiert haben. Junge Autorinnen haben hier zu ihrer eigenen und eigenartigen Sprache als Ausdruck der Befindlichkeit ihrer Generation gefunden. In den betreffenden Texten stehen Frauen im Mittelpunkt, die sich zumeist in Ich-Form artikulieren $^{2}$ und insofern auch eine Stimme - die Stimme ihrer Generation - abgeben. Es handelt sich um Geschichten melancholischen Tenors, die Entfremdung, Einsamkeit und innere Leere der Figuren thematisieren. Dies gilt auch für Jennys Text, der über das Genre des Adoleszenzromans ${ }^{3}$ gleichsam zwei Generationen ins Visier nimmt. Die 68er und die sogenannten Wohlstandskinder ${ }^{4}$ werden in ihrer mentalen Verfassung und vor dem Hintergrund der veränderten Disposition beider Alterskohorten dargestellt. Beachtenswert an dem Roman sind Formen sprachlicher Fixierung für die emotionale Welt der Ich-Erzählerin, die sich in einer poetischen, äußerst plastischen bis synästhetischen, sinnlichen Sprache mitteilt. ${ }^{5}$

1 Alle Zitate aus Jennys Roman erhalten im Folgenden die Sigle BZ. Jennys Roman entstand ein Jahr vor Judith Hermanns Sommerhaus, später, dem Gründungsphänomen des literarischen Fräuleinwunders (vgl. HAGE 1999:244-246). Weitere Texte der Autorin - Der Ruf des Muschelhorns, 2000; Ein schnelles Leben, 2002; Das Porträt, 2007 - sollen nicht an das Debüt heranreichen (vgl. PECHT 2012).

2 Vgl. Juli ZeHs (2002:380f.) Reflexion über die Dominanz der Ich-Perspektive bei den Jungautoren.

3 Adoleszenz sei hier verstanden als ein „Mit- und Gegeneinander von körperlichen, psychischen und sozialen Prozessen“, als ein „,kulturgeschichtlich verankerter“, ,transitorischer Zustand“ (GANSEL / ZIMNIAK 2011:10f.). THOMAS ANDRE (2011:184) bezeichnet diese Schwellen- bzw. Übergangserfahrung als coming-of-age.

4 Vgl. ANDRE (2011). Zu Blütenstaubzimmer vgl. ANDRE (2011:183-193).

5 Vgl. Gesing (2001:334). In diesem Sinne auch ANDRE (2011, Anm. 339:184f.). Die Ich-Erzählerin erfährt „Identität als Austausch der eigenen Innenwelt mit der umgebenden Dingwelt. Jos soziale Energien sind richtungslos: sie besetzen Räume, Dinge, Gedanken.“

FETZ (2003:21f.). Fetz empfiehlt allerdings eine kritische Lektüre der „empfindsamen" Texte Jennys. 
Die adoleszente Hauptfigur Jo wird in zwei Lebensphasen porträtiert, was in der Zweiteilung des Romans seine Entsprechung hat. Ihre Kindheit ist von einem nicht intakten Familienleben überschattet. Nach der Trennung der Eltern lebt sie beim Vater, die Sonntage verbringt sie bei der Mutter, bis diese eines Tages mit einem neuen Partner verreist und der Kontakt für zwölf Jahre abbricht. Nach dem Abitur fasst sie den Mut, mit ihrer Mutter zu telefonieren und sie in einem italienischen Provinzort zu besuchen, wo sie zu dieser Zeit mit ihrem Partner lebt. Mit aller Bestimmtheit setzt sie ihre ganze Energie dafür ein, endlich ein Teil des Lebens ihrer Mutter zu werden $(B Z, 55)$ : sie habe „Haken nach ihr ausgeworfen“ $(B Z, 22)$, ihre Geräusche zu „konservieren“ $(B Z, 47)$ versucht. Doch die Erzeugerin, die signifikanterweise nur im ersten Teil „,meine Mutter“, im zweiten, adoleszenten Teil dann konsequent nur noch Lucy genannt wird, verweigert ihre Mutterrolle und tritt nach außen als Jos ältere Schwester auf $(B Z, 45,53)$. Jo muss immer wieder ihrem feindlichen, harten Blick und ihren abweisenden Gesten begegnen $(B Z, 36,46)$. Das Schlüsselerlebnis für beide Frauen, das allerdings nichts an ihrem Verhältnis zueinander ändert, stellt Lucys katatonische Trauer nach dem Unfalltod ihres Partners Alois dar. Mit diesem Ereignis hängt die zentrale Metapher des Romans, die Blütenstaubmetapher, zusammen. Bereits in der letzten Begegnung mit der Mutter vor deren Abreise wird diese Metapher antizipiert:

Sie sagte, dass sie einen Mann, Alois, getroffen habe, den sie liebe, so wie sie einmal meinen Vater geliebt habe, und dass sie mit ihm fortgehen werde, für immer. Überall, wo ich hinsah, waren diese gelben und roten Blütenköpfe, die einen Duft ausströmten, der mich schwindlig und müde machte. $(B Z, 15)$

Während die Mutter weiterredete, sah Jo den Himmel, der ,wie eine greifbare blaue Scheibe“ über ihnen beiden schwebte. $(B Z, 16)$ Dieser kurze Auszug macht die Poetik des Textes deutlich: Er changiert nämlich zwischen einem emotionslosen Bericht und einem Bild, das symbolisch aufgeladen und zum Träger der Emotion wird. Wie die bunten Blütenköpfe den Verlust symbolisieren, wird die ,blaue Scheibe ' des Himmels zu einem Zeichen der Entfremdung, die die Grundkonstitution beider Frauen ausmacht. Mit der Glassymbolik spielt Jenny, so die hier vertretene These, die im Folgenden zu belegen sein wird, auf SYLVIA PLATHs Ich-Roman mit autobiographischen Zügen Die Glasglocke $(1963)^{6}$ an. Vorerst aber zurück zur Blütenstaubmetapher: Das Motiv taucht erneut auf, als Lucy nach Alois' Beerdigung einige Tage lang morgens Blütenköpfe einsammelt, ins Blütenstaubzimmer, sprich in Alois'

6 Alle Zitate aus Plaths Die Glasglocke erhalten im Folgenden die Sigle GG. 
nun leergeräumtes Atelier geht und den Staub herunterschüttelt, bis er den Boden ganz bedeckt. Dann schließt sie sich im Zimmer ein. Der Staub bildet einen Ersatz für die Asche des toten Alois, dessen Überreste in Folge des Unfalls nicht zurückgeblieben $\operatorname{sind}^{7}$, so dass er nur ein symbolisches Grab unter den Pappeln im Garten bekommt. Lucy inszeniert ein Grab, in dem sie ihm, gemäß der hinduistischen Tradition, in den Tod folgt. So wird das Blütenstaubzimmer zu einer Verschmelzungsphantasie, zu einem symbolischen Scheiterhaufen, auf dem die beiden eine mystische Symbiose eingehen. Durch Jos Eingreifen wird Lucys psychische, lebensverweigernde Blockade gelöst, ihre Verdrängungsstrategien werden dadurch aber nicht angegriffen. Sie weigert sich, über Vergangenes zu reden, will keine Witwe spielen und meint, mit „der Geschichte“ fertig zu sein. $(B Z, 33)$ Jo registriert zahlreiche "Spuren, die von Lucys Versuch zeugen, ihr Gedächtnis totzustellen“ $(B Z, 38)$, zumal sie vermutet, dass es sich bei Alois' Tod nicht um einen Unfall handelte. ${ }^{8}$ Lucys Haltung demaskiert sie als illusorisch: „Als ob es möglich wäre, das Leben eines Menschen auszulöschen, indem man dessen Habe vernichtet und das eigene Bett verschiebt, nur um nicht auf die Bäume zu blicken, die bei seinem Grab stehen." $(B Z, 38)$ Lucys neurotisches Verhältnis zur Vergangenheit und das Bedürfnis, sich durch den Männerblick zu definieren, verweist auf ihre Selbstentfremdung und erklärt die Unfähigkeit, ihre Mutterschaft zu realisieren. Letzteres belastet Jo enorm, was sich in einer Reihe von Zeichen manifestiert. So nennt sie Lucy nach dem Tod von Alois „das Mutterbündel“ $(B Z, 24)$ und bemuttert sie ihrerseits. In ihrer Verlassenheit bezweifelt sie sogar die biologische Mutterschaft Lucys und schließt das Muttermal neben dem Bauchnabel mit einer Phantasie der eigenen Leiche zusammen. $(B Z, 108)$ Um die Mutter festzuhalten, möchte sie sie krank und in einer Anstalt - d.h. immer zugänglich - erleben. Dabei ist sie nur im Traum erreichbar und selbst da in Form einer Todesphantasie. ${ }^{9}$ In Wirklichkeit umgebe Lucy „eine Leichtigkeit [...] wie eine undurchdringliche Glocke“ (BZ, 67). Spätestens an dieser Stelle sind die Parallelen zu Plath eindeu-

$7 \quad$ „Von Alois ist kein Körperteil zurückgeblieben. Und keine Asche. Die wurde unmittelbar danach vom Wind fortgetragen. Geschleudert und explodiert.“ (BZ, 53)

8 „Wenn du schon nicht sagen kannst, ob es Vito war, der das halbe Dorf hat abreißen lassen, um seine Hotels zu bauen, könntest du mir jetzt wenigstens erzählen, was mit Alois geschehen ist. Es war doch kein Unfall, nicht wahr?“" $(B Z, 67)$

9 Jo sieht sie in einem weißgekachelten Badezimmer sich die Zehennägel lackieren. Doch als sie ihre Hand auf ihren Kopf legen möchte, bemerkt sie ihren nackten Schädel. $(B Z, 67)$ 
tig. Sie kulminieren im Zusammenhang mit Lucys abschiedslosem Verschwinden mit ihrem neuen Partner, der bezeichnenderweise Vito heißt und also eine Art vitalisierende Pille für Lucy darstellt. Sie verreisen an ihrem 45. Geburtstag auf eine Insel im Indischen Ozean, womit Lucy ihre Tochter endgültig zurück und im Stich lässt. Ihre Gefühlskälte und Rücksichtslosigkeit gegenüber Jo erreichen damit einen Höhepunkt und stürzen diese in eine tiefe Krise, die Jenny über verblüffend viele Parallelen zu Plaths Glasglocke plausibel zu machen versucht und damit eine latente Verwandtschaft zur amerikanischen Dichterin und deren jungen Frauenfiguren zum Ausdruck bringt. Plath (1932-1963) war bekanntlich eine Ikone der 68er Generation in Amerika und gilt nach wie vor als Symbolfigur der Frauenbewegung. ${ }^{10}$ Seit früher Jugend litt sie an bipolarer Störung, an der sie schließlich zerbrach, indem sie sich das Leben nahm. In ihren Dichtungen, Tagebüchern und dem erwähnten Roman dekonstruiert sie amerikanische Mythen. In der Glasglocke demaskiert sie das Image einer glücklichen, erfolgreichen, heilen amerikanischen Familie sowie das Bild eines satten und selbstzufriedenen Staates als Fassade, hinter der sich tiefe Verstörungen und Deformationen verstecken (BRONFEN 1998:100f.). Im Mittelpunkt des Romans steht die Identitätsentwicklung Esther Greenwoods. Die neunzehnjährige Ich-Erzählerin sieht sich fragwürdigen, beengenden bis repressiven Normen, Konventionen und Denkweisen konfrontiert, denen gegenüber sie eine ambivalente Haltung entwickelt, da sie als angehende Dichterin auf Anerkennung angewiesen ist. Die Glasglocken-Metapher gilt inzwischen als Topos der als traumatisch erlebten Einsamkeit und Entfremdung. Neben der Titelmetapher benutzt Plath in ihrem Roman eine Reihe von verwandten Metaphern wie Glaswände, Flaschen oder andere Gefäße aus Glas, um die psychologische Isolation der Protagonistin auszudrücken. ${ }^{11}$ Auf eben diesen Topos rekurriert Zoë Jenny, indem sie zu-

10 Der Roman Die Glasglocke wurde als ein feministisches Manifest gefeiert, das doppelte moralische Standards und falsche Versprechen gegenüber den in den 1950er Jahren in Amerika geborenen Frauen aufdeckt (vgl. BRONFEN 1998:125). Es gibt zudem textuelle, psychoanalytische und kulturelle Parallelen zwischen Plaths Werk und dem Virginia Woolfs sowie zwischen dem Schaffen dieser beiden Autorinnen des 20. Jahrhunderts und dem ihrer literarischen Mutter Charlotte Brontë. (BRAIN 2001:141)

11 Ihnen allen ist eigen ,their aspect for doubly-felt isolation, for the entrapped text and the entrapped self are present among people and life's events, but they are unable to speak to and to reach out to them or be touched by them. This isolation is particularly painful because the poet can clearly see and observe every detail, but still she remains cut off from the world. "BLOSSER (2001:220) 
nächst Lucys Befindlichkeit mit der Metapher einer undurchdringlichen Glocke beschreibt. Auch in Plaths Roman ist die Mutter eine relevante, wenn auch nicht so zentrale Figur wie bei Jenny. Und dennoch klingt die Litotes bei Plath beredt: „Meine eigene Mutter war keine große Hilfe.“ $(G G, 46)^{12}$ Da die Glocken-Metapher bei Jenny die Perspektive Jos beschreibt, ist sie und nicht Lucy das eigentliche Opfer einer gestörten Wahrnehmung, die sich in plastischen Bildern ausdrückt, die ihre Innenwelt bestimmen. Seit der Kindheit leidet sie an einer Insektenphobie, die mithin ein frühes Zeugnis ihrer besonderen Sensibilität darstellt. Wegen eines Spinnengewebes zu Hause beim Vater sei die Nacht, so die Ich-Erzählerin, „nur manchmal draußen“ geblieben. (BZ, 7f.) Eine Reminiszenz der Kindheitsängste stellen die Skorpione im italienischen Haus der Mutter dar: „Dort, im Innern der Decke, leben die Skorpione, Hunderte vielleicht. Sie fressen, schlafen und paaren sich, alles direkt über meinem Kopf, und ab und zu geht einer von ihnen auf meinen Wänden spazieren." $(B Z, 97)$

Jo wird ferner von Alpträumen geplagt, in denen sie immer wieder ihr Leben retten muss. $(B Z, 33)$ Immer stärker treten im Roman die Figuren des Todes in den Vordergrund. Dies geht mit Jos wachsendem Bewusstsein einher, bei der Mutter - und damit wohl auch bei sich selbst - nicht ankommen zu können. Der einschneidende Moment hierfür ist signifikanterweise wieder an Plaths Roman angelehnt. An Lucys 45. Geburtstag erinnert sich Jo, dass diese einmal gesagt habe, ein Garten sei erst dann richtig schön, wenn, neben der Treppe, die in den Garten führt, ein Feigenbaum stehe. In einem Pflanzengeschäft kauft Jo auch einen; der Baum hat die Größe eines Haselstrauches, die Früchte sind noch klein und hart: „Über dem Stamm, schwächlich wie ein abgemagertes Bein, wachsen die Feigenblätter nach allen Seiten hin.“ $(B Z, 68)$ Jo stellt sich vor, dass Lucy am nächsten Morgen mit Freude die Überraschung entdeckt. Doch Lucy ist inzwischen mit Vito über alle Berge: Der Postbote bringt am nächsten Morgen eine Karte mit der Luftaufnahme einer Insel im Indischen Ozean mit der Botschaft, Lucy wolle die Gelegenheit nutzen, „einmal richtig auszuspannen“ (BZ, 71). Das Feigenbaum-Motiv

12 Sylvia Plaths Mutter, Aurelia Plath, kommentierte das Karikaturhafte der Mutter-Figur im Roman folgendermaßen: Die Glasglocke und einige Gedichte ,involve the mother figure as the whipping boy, so characteristic of the Fifties" (GILL 2008:81). Neben der leiblichen Mutter gibt es in Plaths Roman verschiedene andere Mutter-Figuren, an die das regulative Recht der Kultur geknüpft ist. BRONFEN (1998:113). 
taucht zum Ende des Textes noch einmal im Zusammenhang mit dem Jahreszeitenwechsel auf:

Der Stamm des Feigenbaums stülpt sich wie ein Knorpel aus der harten Erde. Kleine knittrige Früchte hängen an ihm. Die Blumen sind allesamt braun verdorrt. Die Luft riecht nach Schnee. Im Blütenstaubzimmer liegt noch das Laken, auf dem Lucy gelegen hat. Ich kann mich an einen bestimmten Geruch erinnern, der immer in diesem Raum hing, aber die Kälte hat jetzt alle Gerüche im Haus vertrieben. $(B Z, 113)$

In der Glasglocke steht das Feigenbaum-Motiv in direktem Zusammenhang mit der Befindlichkeit der Hauptfigur. In dem von der Ladies Day-Redaktion zugeschickten Geschenkbuch findet Esther eine Liebesgeschichte, die folgendermaßen beginnt: „Dieser Feigenbaum stand auf einer grünen Wiese zwischen dem Haus eines jüdischen Mannes und einem Kloster, und regelmäßig trafen sich der jüdische Mann und eine schöne, dunkelhäutige Nonne unter dem Baum, um die reifen Feigen zu pflücken.“ $(G G, 62)$

In diesem ersten Textabschnitt repräsentiert der Feigenbaum vor allem die frustrierte Sehnsucht, aber Plaths Ich-Erzählerin interessiert sich nicht so sehr für die Liebesgeschichte als für die Qualitäten des Baums. Ihr gefällt „,vor allem der Teil über den Feigenbaum im winterlichen Schnee und der Feigenbaum im Frühling mit all den grünen Früchten.“ $(G G, 62)^{13}$ Doch die eigentliche Symbolik der Pflanze entwickelt die Autorin erst im folgenden Abschnitt:

Ich sah, wie sich mein Leben vor mir verzweigte, ähnlich dem grünen Feigenbaum. Gleich dicken, purpurroten Feigen winkte und lockte von jeder Zweigspitze eine herrliche Zukunft. [...]. Ich sah mich in der Gabel dieses Feigenbaumes sitzen und verhungern, bloß weil ich mich nicht entscheiden konnte, welche Feige ich nehmen sollte. Ich wollte sie alle, aber eine von ihnen nehmen bedeutete, alle anderen verlieren. $(G G, 85)$

Die Feigen repräsentieren verschiedene Lebenswege: ein Zuhause mit Ehemann und Kindern, den Ruhm als Dichterin, die Universitätskarriere, die Karriere als Redakteurin, Reisen, eine Reihe von Liebhabern, olympische Medaillen. Die Feigen verdorren, während Esther sich für keine der Möglichkeiten entscheiden kann. Letztendlich verweigert sie jede Entscheidung und erstarrt, oder zieht sich - wenn man so will - unter die Glasglocke zurück. Der Feigenbaum wird bei Plath also zum Sinnbild ungenutzter Potentia-

13 „Tellingly, it is not the romantic plot that attracts Esther's interest. What she is seduced by are the aesthetic qualities of the tree - its solidity and abundance." (GILL 2008:76). 
le und zum Zeichen der Lähmung, die mit dem Ideal der Weiblichkeit der 50er Jahre in Amerika erklärt werden kann. Die junge Frau leidet, weil sie so viel sein möchte in einer Welt, die den Frauen kaum etwas zu sein bzw. zu werden erlaubt. (GILL 2008:76) Zugleich versinnbildlicht der Baum Fülle und Überfluss im Gegensatz zum Mangel. ${ }^{14}$

In Jennys Text verweist das Feigenbaum-Symbol auf eine ähnliche polare Struktur: Während der junge Feigenbaum für Jo Heimat, Zugehörigkeit und Geborgenheit repräsentiert, wird er, in der Kälte erstarrt, zum Sinnbild des Mangels, des emotionalen Hungerzustands der Protagonistin. Die universale Symbolik des Lebensbaums realisiert sich somit hier als ein beunruhigendes Negativ der Vitalität und Gesundheit. Wie in der bei Plath zitierten Liebesgeschichte grenzt Lucys Haus bezeichnenderweise an ein Kloster, an dessen Mauer Alois bald seine Ruhestätte bekommen soll: „Immer am Abend kommt ein Mönch auf die Loggia des Klosters und gießt die Geranien. Das Wasser tropft mit einem leise klatschenden Geräusch aus den Blumenkisten auf die Straße hinunter, die das Kloster von unserem Garten trennt.“ (BZ, 64)

Noch bevor der Postbote mit Lucys unverhoffter Nachricht erscheint, lässt Jenny ihre Ich-Erzählerin einem Todesboten begegnen. Am Gartentor auf Lucy wartend trifft sie zwei Jungen, die sie dazu nötigen, ihnen tote Schmetterlinge abzukaufen. Das Motiv der toten Schmetterlinge tauchte in einer ähnlichen Konstellation auch schon früher auf: Eines Tages führt Lucy ihre Tochter in den Wald an einen Fluss, wo diese todeslustige Schmetterlinge zu retten versucht, indem sie sie einen nach dem anderen aus dem Wasser holt. Dies ist vergeblich, denn sobald ihre Flügel wieder trocken sind, fliegen sie zurück ins Wasser, ,in den Tod. [...] Kein einziger Schmetterling ist zurückgeblieben, alle haben sich den Wasserfall hinuntergestürzt.“ (BZ, 37). In diesen beiden Szenen, wie schon in Jos Alpträumen, laufen Fäden zusammen, die diese, ihre Mutter und den Tod kurzschließen. Auch die wiederholte Erfahrung des feindlichen Blicks und der ablehnenden Haltung Lucys parallelisiert die Autorin mit Metaphern des Todes, die, unter Rückverweis auf Plaths Glasglocke, auf die suizidale Disposition der Ich-Erzählerin schließen lassen. Jo verfällt immer mehr einer psychischen Lähmung: „Hier sollte ich liegenbleiben, denke ich, und werden wie dieser Stein [...] irgendwann wür-

14 „The second point is that the images of self as starving because she is too greedy to select just one fig sustain a major concern in the novel - about need and satiety, excess and restraint. The reverse side of such metaphors, $[\ldots]$ emerges in images of evacuation, poison sickness and bile." (GILL 2008:77). 
de es vollkommen still sein, Moos über mich wachsen." $(B Z, 35)^{15}$ Nach dem Verschwinden der Mutter verdichtet sich die Todesmetaphorik zunehmend. Einmal formuliert Jo ihre Vorstellung, dem Tod nahe zu sein, direkt: Im Jahre 2000 soll sich das Nachbardorf in einen berühmten Wallfahrtsort verwandeln: „Falls ich bis dahin überleben sollte, werde ich vierundzwanzig sein.“ $(B Z, 25)$ Jo phantasiert über ihren toten Körper, der im Autopsieraum auf einer hohen Bahre liegt $(B Z, 108)$, oder sie stellt sich vor, aus lauter Angstkugeln zu bestehen:

Ich bin voll mit diesen Kugeln, die mich von innen ausbeulen und verformen, so dass ich in alle Richtungen auseinanderzubrechen drohe. Jede einzelne dieser Kugeln ist ein selbständig funktionierender Organismus. Sie bekriegen sich gegenseitig, denn jede will mich ganz. Die Lucy-Angstkugel ist die größte, manchmal verschwindet sie, aber jetzt ist sie wieder aufgetaucht und wächst und bekriegt die anderen. $(B Z, 109)$

Die Entfremdungserfahrung der Ich-Erzählerin manifestiert sich in einer schwachen Ich-Grenze. Die Außenwelt füllt in Form von fremden Introjekten das Innere der Figur aus, was sie körperlich intensiv als Gefahr des Auseinanderbrechens empfindet. Die Metaphern der Desintegration werden vielfach variiert: Auf Lucys Party erlebt sie sich als ,eine auseinanderbröckelnde Statue“. (BZ, 27) Auf einer Rave-Party sieht sie im Licht des Stroboskops „nur noch einzelne Körperteile“ und erlebt sich als „Teil einer großen Körpermaschine, die zittert und die sich aufbäumt und einen hysterischen Lärm veranstaltet, gegen die schreckliche Stille im Kopf" $(B Z, 93)$. Wie in der Glasglocke wird bei Jenny der One-Night-Stand zu einer Erfahrung der Entfremdung: Das Gesicht des Partners ist in Bruchstücke auseinandergefallen, Jo starrt an ihm vorbei an die Decke, vernimmt Geräusche aus der Nachbarwohnung und hält den Atem an, während ihr Arm unbequem gekrümmt „,wie etwas Abgetrenntes auf der Matratze [liegt]“ (BZ, 86). Während Plaths Protagonistin Esther wegen einer starken Blutung ins Krankenhaus muss, wird sich Jo einer Abtreibung unterziehen müssen. Danach fühlt sie sich davonrollen wie ,eine glatte bleierne Kugel“ ( $B Z, 88)$. Jos depressiver, mit mangelnder Selbstreflexion einhergehender Zustand manifestiert sich auch in einer Sprachkrise. Hier rekurriert Jenny abermals auf Plath, indem sie ihrer Figur den letzten Schutz - den Schutz der Wörter $-{ }^{16}$ entzieht: „Früher konnte ich

15 An einer anderen Stelle erscheint Lucy ihrer Tochter ,wie ein einziger großer Stein, ihr Blick brennt auf meinem Kopf, aber ich wage nicht, ihr in die Augen zu sehen, die klein und hart über diesem Flügel [ihres Rockes] sitzen“ (BZ, 47).

16 „Eine Wand aus Wörtern, die mich umgab und schützte, solange ich las, und ich tat nichts anderes. [...] Ich las und war ein Schiff auf Reisen.“ (BZ, 42f.). 
durch die Wörter gehen wie durch offene Türen. Jetzt stehe ich davor, und nichts geschieht.“ $(B Z, 44)$ Diesen Zustand, nicht mehr den Worten und ihrer Bedeutung folgen zu können, beschreibt sie als den Zustand des Ausgeschlossen- oder Abgeschnittenseins (BZ, 43f.), dem sie immer mehr erliegt. Die Erfahrung der Desintegration - etwa in Form des als fragmentiert erlebten Körpers und der Sprachkrise - teilt Jennys Protagonistin mit anderen Frauenfiguren der Gegenwartsliteratur, insbesondere des literarischen Fräuleinwunders. ${ }^{17}$ In Anlehnung an Die Glasglocke lässt Jenny ihre Protagonistin die metaphorische Dunkelheit erfahren. Esther kam es vor, ,als würde [sie] tiefer und immer tiefer in einen schwarzen Sack gestopft, ohne Luft und ohne Entkommen" ( $G G, 140)$. Das Gefühl, in die Enge getrieben zu sein und nicht mehr leben zu wollen, resultiert in der Glasglocke bekanntlich in Esthers Suizidversuch, als sie eine Menge Pillen schluckt und sich in einem Erdloch im Hauskeller verkriecht: „Es dauerte eine Weile, bis ich meinen Körper in die Öffnung gehievt hatte, aber schließlich gelang es mir, und nun kauerte ich wie ein Kobold am Eingang der Dunkelheit.“ $(G G, 183)$

Jenny variiert, in evidenter Anlehnung an Plath, die Metaphern der Enge, die den Tod und den pränatalen Zustand kurzschließen, um die regressive Tendenz der Ich-Erzählerin plausibel zu machen. Schlaflose Nächte quälten Jo schon immer, jetzt bringt aber die Helligkeit des Tages nicht mehr das Gefühl, gerettet zu sein. $(B Z, 77)$ Im Gegenteil: Sie entwickelt eine Lichtüberempfindlichkeit, die sie dazu bringt, sich vor dem Licht zu verstecken. Unter der Decke versteckt denkt sie sich Höhlen aus:

Höhlen, die sich hinter einer winzig kleinen Öffnung auftun, da wollte ich hineinschlüpfen, eine atmende Kugel werden, dort drinnen, wo kein Licht, kein Laut, kein panisches Leben geboren werden kann. [...] Das Bett, in dem ich liege, ist eine Insel, die drauf und dran ist unterzugehen. $(B Z, 107)$

Auch ihr Schlafsack erscheint ihr als „eine lange dunkle Röhre“, in die sie hineinkriecht, während der Winter ins Haus dringt: „Es ist vollkommen dunkel in der Röhre, und ich höre das Pochen des Blutes in den Ohren. In diesem Pochen und dem Atem, der die Luft in der Röhre erwärmt und einen Kokon bildet, wünschte ich einzuschlafen und wegsterben zu können." (BZ, 112f.)

17 Vgl. Gustafson (2007: insbesondere 1, 3f. und 13-15). Gustafson stützt ihre Analyse auf Julia Kristevas Black Sun: Depression and Melancholia (1989), wo diese erläutert, wie Melancholie und Depression eine „Krise des Denkens und Sprechens“ sowie eine „Krise der Repräsentation“ konstituieren. 
Die depressiven Züge der adoleszenten Jo verstärken sich, nachdem sie ihre Fluchtträume aufgeben ${ }^{18}$ und einsehen muss, dass sie nun endgültig alleine dasteht. Diese Erkenntnis „platzte in ihrem Kopf wie ein Wassersack“ $(B Z, 102)$. In einer der letzten Szenen geht sie ,wie auf einem Faden, der [sie] langsam, Schritt für Schritt aufwickelt" (BZ, 121). Zuletzt scheint sie wortwörtlich zu erfrieren: Es fällt der erste Schnee, aber sie sei ja „schon so lange in der Kälte“, dass sie sie langsam vergesse. Sie setzt sich auf eine Parkbank und verharrt in einem Zustand der Erstarrung: Sie werde hier warten "auf die weiße Schicht über dem Boden. Auf die Decke aus Schnee“ $(B Z, 122)$. Es ist nur allzu selbstverständlich, dass Jenny hier noch einmal auf Plaths Roman rekurriert. Er endet mit einer Art Wiedergeburt Esthers: Der Ärzteausschuss wird sie mit großer Wahrscheinlichkeit aus der Anstalt entlassen. Nach sechsmonatiger Unterbrechung soll sie das Leben wieder da aufnehmen, wo sie es zuvor unterbrochen hat. Auch sie scheint einem Faden zu folgen: „Alle diese Augen und Gesichter wendeten sich mir zu, und indem ich mich von ihnen wie von einem Zauberfaden lenken ließ, betrat ich den Raum." $(G G, 262)$ In Plaths letztem Kapitel deckt frischer Schnee das Gelände der Anstalt. Esther sinniert über ihre Vergangenheit, die sie, zu ihrem Leidwesen, nicht wie einen bösen Traum abtun kann: „Vielleicht würde das Vergessen wie eine freundliche Art von Schnee sie alle [ihre Erinnerungen] erstarren lassen und zudecken. Aber sie waren ein Teil von mir. Sie waren meine Landschaft." $(G G, 254)$

Die junge Frau kann sich nicht sicher sein, ob sich nicht eines Tages „die Glasglocke mit ihren erstickenden, lähmenden Verzerrungen“ wieder über sie senken würde. $(G G, 258)$ Die Freiheit, die Esther möglicherweise bevorsteht, ist also von einer Angst und Unsicherheit überschattet, die aus der traumatischen Erinnerung und Einsicht resultiert. Auf dieses Grundgefühl färbt nicht zuletzt der völlig unerwartete, fast absurd scheinende Suizid Joans ab, die sich scheinbar längst auf dem Weg der Besserung befand. Esther geht zur Beerdigung und fragt sich dabei, was sie selbst da eigentlich zu Grabe zu

18 Ferne stellt die Projektionsfläche für Wünsche und Sehnsüchte der Figuren dar: Bereits in der Kindheit imaginiert Jo, mit ihrem Vater auf der Flucht zu sein. $(B Z, 43)$ In Italien träumt sie davon, mit ihrer Freundin Rea nach Milwaukee auszuwandern, dann mit dem Freund Luciano „fortzugehen“ $(B Z, 110)$. Doch dieser hat seine Träume und sich selbst inzwischen aufgegeben und arbeitet nun auf einer Baustelle. Auch die neue Familie des Vaters erlebt Jo nicht als ihr Asyl. Das Fernwehmotiv - ein Topos der Schweizer Literatur - mag bei Jenny ein subtiler Träger der Kritik an der Heimat sein. Vgl. HeFFERNAN (2010:285, 288). 
tragen glaube. Das tiefe Loch in der Erde erscheint ihr als eine Wunde, die der nächste Schnee bald verschließen wird. ${ }^{19}$ Mit äußerster Mühe behauptet Esther allem zum Trotz ihre Existenz, ihr neu gewonnenes, hart erkämpftes Selbst: „Ich bin, ich bin, ich bin.“ $(G G, 260)$ Die Symbolik des Textes scheint also an dieser Stelle eine andere, und zwar tragische Botschaft über die psychische Fragilität zu vermitteln, als die Protagonistin mit der Vorstellung ihrer Wiedergeburt wahrhaben will. ${ }^{20}$

Mit Blick auf Plaths Roman stellen sich die Anzeichen für eine Loslösung und einen Neuanfang der adoleszenten Protagonistin in Blütenstaubzimmer als äußerst schwach dar. ${ }^{21}$ Die gesamte Poetik des Textes spricht im Gegenteil die Sprache der Dissoziation. Jos Orientierungslosigkeit und ein tiefes Bedürfnis nach Geborgenheit relativieren den vermeintlichen Faden, den das Ich zuvor gefunden zu haben scheint. Auch der Schnee verweist in seiner Eigenart auf die Vergänglichkeit des Lebens und suggeriert unter Rückbesinnung auf die Topoi der Schweizer Literatur den Schnee- bzw. Kältetod (vgl. HEFFERNAN 2010:289-291, hier 291).

Jenny stellt ein tragisches Bild des bindungsunfähigen bzw. bindungslosen Einzelnen dar, der weder in anderen noch in sich selbst Halt findet und ein vererbbares Trauma des Deplaziertseins in und mit sich herumträgt. Während Esther Greenwood im Sinne der Feigenbaum-Metapher mit Identitäten experimentiert und zum Schluss zu einer eigenen findet ${ }^{22}$, bewegt sich Jo in einem geistigen Vakuum, in einer trostlosen Welt, in der ihr keine akzeptablen Identitätsangebote zur Verfügung stehen. Mit anderen Worten: Sie kann die Vielzahl von Rollenangeboten, die als Signum des modernen Lebens gilt (MEHRFORT 2006:204f.), gar nicht erst wahrnehmen, da sie durch die Glasglocke nur ein deformiertes Bild aufnimmt. Jos Zustand stellt sich als eine weibliche

19 „Irgendwo dort war ein schwarzes, zwei Meter tiefes Loch in den harten Boden gehackt worden [...] die eigentümlich gelbe Erde unserer Gegend würde die Wunde in all dem Weiß verschließen, und der nächste Schnee würde auch die Spuren von Neuheit an Joans Grab auslöschen." $(G G, 260)$.

20 So auch Ted Hughes in Sylvia Plath and her Journals (1982). Vgl. BRONFEN (1998:114f.).

21 Da wäre die quasi delegierte Kindheit (sie schenkt ihre Kinderschuhe ihrem Halbbruder/ihrer Halbschwester, der/die bald auf die Welt kommt) und die autonome Entscheidung, wegzugehen, statt sich zähneknirschend an die neue Familie des Vaters anzupassen.

22 „It is, as if [...] she were able to construct the imaginary home she can now successfully locate herself in." BRONFEN (1998:123). 
Form des ,aquarium life' dar - als ein Leben, das keinen Sinn zu bieten scheint und sich in einer unterschwelligen Depression manifestiert. Auf diese Weise ist die Beschaffenheit des postmodernen Subjekts - Orientierungslosigkeit, Ich-Schwäche, Profillosigkeit, tiefe Sinnkrise - eindeutig formuliert. ${ }^{23}$ Die Aquarium-Metapher gilt für viele Protagonistinnen der jungen deutschsprachigen Literatur von Frauen (GUSTAFSON 2007:1, 19). Trotz der nach innen gekehrten Revolte der Protagonistin Jo (FETZ 2003:21) geraten gesellschaftliche Hintergründe und Determinanten stark in den Fokus der Autorin, insbesondere die Frage nach dysfunktionalen Familien bzw. Beziehungen um die Jahrtausendwende. In einem Interview macht die Autorin unmissverständlich die etablierte ältere Generation - die 68er - verantwortlich für den Verrat an ihren ehemaligen Werten und damit für den gegenwärtigen, desolaten Zustand der Gesellschaft - ihre Seelenverkümmerung, Gleichgültigkeit, Beziehungslosigkeit. Ihre, Jennys Generation, sei deswegen „stumm“, ihr sei „Vergeblichkeit des Kampfes um eine bessere Welt“ immanent. ${ }^{24}$ Diese Diagnose lässt sich auch im Roman leicht nachweisen. Die Krise erfasst hier beide Generationen, Frauen und Männer gleichermaßen, wobei defizitäre Kinderpersönlichkeiten als direkte Konsequenz auf ebensolche Elternpersönlichkeiten zurückzuführen sind ${ }^{25}$ Und so sind Lucys Lebensgefährten für Jo genauso unerreichbar wie diese selbst: Solipsistisch auf sich selbst bezogen eignen sie sich kaum für die Vaterrolle. Dieses Unvermögen steht in keinem Zusammenhang mit dem Bildungsstand oder der Lebenseinstellung. Denn in dieser Hinsicht repräsentieren sie unterschiedliche Typen: Als Maler steht Alois zwar für Kreativität und Bildung, doch Jo entgeht nicht, dass er möglicherweise seinen Unfalltod selbst herbeigeführt hat. Im Haus hängen drei seiner Bilder. Sie alle stellen immer nur ein Motiv dar: den von einem Blitz gespaltenen Baum, der offenbar die Innenwelt des Künstlers spiegelt und seine Frage plausibel macht, ob ein Haus wie ein alter

23 Jenny riskiert damit keine neue These. Vgl. ANDRE (2011:245-250) sowie FETZ (2003: insbesondere 17, 21-24).

24 „Wenn man sich die Elterngeneration - die sogenannten 68er - heute anschaut, denkt man doch: Da hat sich die Welt aber schnell umgekehrt. Wirklich rasant. Der Verrat an den besseren Werten, die damals gepredigt wurden, ist schon enorm. Da muss die nächste Generation doch fragen: Ist das eine Riesenverarschung oder was?"“ JENNY (2002:84).

25 ThOmAs ANDRE (2011:186f.) spricht in diesem Zusammenhang vom ,psychischen Energietransfer, der zwischen den Generationen stattfindet. [...] Für die emotionale Ökonomie des Elternteils bezahlt das Kind mit einem Verlust an Energie." 
Mensch plötzlich in sich zusammenbrechen könne. (BZ, 48, 54) Vito, der neue Partner Lucys, stellt ein Pendant zu Alois dar. Wie sein sprechender Name hat er sich mit dem Leben gut arrangiert: Er ist ein „ungeheuer beschäftigter" Bauunternehmer und Hotelier, der Inbegriff des rücksichtslos schreitenden Neuen, der ,nur eingepflanzten, satt gewordenen Zufriedenheit“". $(B Z, 50)$ Keiner der 68er ist der Sensibilität Jos gewachsen, keiner reif genug, sich auf sie einzulassen, sie als Tochter wahrzunehmen. Das Porträt der Gleichaltrigen gerät nicht positiver und beunruhigt dadurch nicht weniger als das der Elterngeneration. Da ist einmal Jos Freundin - die pragmatische, berechnende, undurchlässige und ich-bezogene Rea, die ,vorwiegend in Stichworten spricht“ und an ,irgendeine gemeine Figur aus einem Zeichentrickfilm“ erinnert (BZ, 76f.). Ihr zur Seite steht der gewalttätige und, wie sich erweist, im wahrsten Sinn des Wortes ,irre Typ“ Nicola $(B Z, 88,99 f$.). Den Kreis schließt Kurt Cobain-Fan Luciano, der ,wie ein gefährdetes kleines Tier“ aussieht, „das zu erschöpft ist, ein Versteck zu suchen“ $(B Z, 115)$. So stellen sich die Figuren in Blütenstaubzimmer allesamt als lose Monaden dar: als bindungsunfähig, narzisstisch und gefühlskalt. Dieses skeptische Bild wird raumdramaturgisch durch unheilvolle Landschaften unterstützt. Natur und Architektur inszeniert Jenny gleichsam als feindlich. Der Topos Italien stellt sich als dystopisch dar. ${ }^{26}$ Die Ich-Erzählerin erfährt das Land über unerträgliche Hitze und das damit verbundene Unwohlsein bzw. über die Kälte. (BZ, 36f., 51, 113f., 121) Pappeln und Blütenstaub sowie Insekten fungieren als Figuren des Todes. Das Haus sei für Jo „kein Haus“ $(B Z, 59)$, auch der Garten mit Alois' Grab an der Klostermauer und dem einsamen, frierenden Feigenbaum wird ihr nicht zur Heimat. Die direkte Umgebung erlebt sie als „ein sterbendes Tier“ (BZ, 73f.), als eine durch Fortschritt und Zivilisation degradierte, bedrohliche, vom Baulärm bestimmte, gar apokalyptische Landschaft ohne Bezug zum Subjekt. ${ }^{27}$ Zwischen der Protagonistin und den Repräsentationen ihrer Umwelt kann sich somit keine Kommunikation einstel-

26 „Bin froh, wenn ich bald wieder von hier fort kann. Ist eine hässliche Stadt hier.“ $(B Z, 76)$ „Es muß doch andere Orte geben, überlege ich, ganz andere als diesen hier.“ (BZ, 95).

27 Das Nachbardorf verwandelt sich in eine gigantische Baustelle; die benachbarte Stadt zeichnet sich durch Pisse- und Desinfektionsmittelgeruch, durch ein gigantisches Einkaufszentrum, durch Hotels und Häuser im Rohbau aus. (BZ, 25, 42, 60, 66 und 74) Apokalyptische Motive: in Form des Films Apokalypse Now, dessen Schluss Rea als „ein perfektes Feuerwerk“ immer wieder neu genießt $(B Z, 80)$, und in Form eines Computerspiels, mit dem Jo nicht aufhören kann, bevor sie die Stadt vor stürzenden Meteoriten gerettet hat. $(B Z, 109)$. 
len. Die Antwort des Textes auf die grundsätzliche Frage nach der mentalen Verfassung um die Jahrtausendwende gibt sich als Anklage der Elterngeneration zu erkennen. ${ }^{28}$ Jenny legt eine depressive Prosa vor und multipliziert Anzeichen der Regression ihrer Protagonistin. Über Parallelen zu Plath wird der resignative Ton von Blütenstaubzimmer stärker und dramatischer. In diesem Sinne suggeriert das letzte Bild - Jos Warten auf „eine Decke aus Schnee" (BZ, 122) - eher den Exitus als eine Reife, die das Gegebene hinzunehmen bereit wäre, so dass hier wohl von misslungener Adoleszenz auszugehen ist. ${ }^{29}$ Vor allem aber verrät die Poetik Jennys hier einen latenten Geltungsanspruch: Sie erhebt die Botschaft des Textes auf die Ebene einer universalen Aussage über die gegenwärtige Subjektkonstitution.

\section{Literatur}

ANDRe, ThOmas (2011): Kriegskinder und Wohlstandskinder. Die Gegenwartsliteratur als Antwort auf die 68er. Heidelberg.

Blosser, Silvianne (2001): A Poetics on Edge: The Poetry and Prose of Sylvia Plath. Bern.

Brain, TrACY (2001): The Other Sylvia Plath. Harlow.

Bronfen, Elisabeth (1998): Sylvia Plath. Michigan.

FETZ, BERNHARD (2003): Was ist gegenwärtig an der gegenwärtig neuesten Literatur? Ein Quellenstudium zur Bewusstseinslage am Beispiel von Bettina Galvani, Zö̈ Jenny, Juli Zeh, Martin Prinz und Thomas Raab. In: AsPETSBERGER, FrIEDBERT (ed.): Trends und Motive in der (österreichischen) Gegenwartsliteratur. Innsbruck, 15-35.

Gansel, Carsten / Zimniak, PAWEl (2011): Adoleszenz und Adoleszenzdarstellung in der Literatur - Vorbemerkungen. In: CARSTEN GANSEL / PAWEL ZIMNIAK (eds.): Zwischenzeit, Grenzüberschreitung, Aufstörung. Bilder von Adoleszenz in der deutschsprachigen Literatur. Heidelberg, 9-13.

GESING, FRITZ (2001): Blütenstaub im crazy Faserland. Stimmen der Jugend am Ende des 20. Jahrhunderts. In: Bogdal, Klaus-Michael / GutJAHR, ORTRud / PFEIFFER, JoACHIM (eds.): Jugend. Psychologie - Literatur - Geschichte. Würzburg, 323-350.

28 Eine ähnliche Diagnose etwa in: KRAChT $(1997: 120,146)$. Vgl. hierzu: GESING (2001: insbesondere 330-332); vgl. zudem FeTZ (2003:21).

29 Carsten Gansel unterscheidet drei Typen misslungener Adoleszenz: eingefrorene Adoleszenz (Verdrängungs-, Abwehrmodell), zerbrochene Adoleszenz (Anpassungsmodell) und ausgebrannte Adoleszenz (Weiterwirken früherer Traumatisierungen; GANSEL / ZiMNIAK 2011:34). Der letzte Typ scheint am ehesten auf Jo zuzutreffen. 
Joanna Firaza

GILL, Jo (2008): The Cambridge Introduction to Sylvia Plath. Cambridge.

Gustafson, Susan E. (2007): Asymbolia and Self-Loss: Narratives of Depression by Women in Contemporary German Literature. In: Monatshefte 99/1:1-21.

HAGE, VOLKER: Literarisches Fräuleinwunder. In: Der Spiegel, 22.03.1999:244-246.

HEFFERNAN, VALERIE (2010): Unschweizerische Schweizerliteratur? Ruth Schweikert, Peter Stamm, Zoë Jenny. In: BARKHOFF, JÜRgen / HefFERnAn, VAlerie (eds.): Schweiz schreiben. Zu Konstruktion und Dekonstruktion des Mythos Schweiz in der Gegenwartsliteratur. Berlin/New York, 283-295.

JENNY, ZoË ( 1997 / 1999): Das Blütenstaubzimmer. München.

- (2002): Beim Schreiben muss man sich die Maske vom Gesicht reißen. In: SELCHOw, STEPhANIE VON (ed.): Siehst du den Horizont? Franka Potente, Zoë Jenny et alii. über ihr Leben. Aarau/Frankfurt (M.), 74-84.

KRACHT, CHRISTIAN ( 1995 / 1997): Faserland. Köln.

MEHRFORT, SANDRA (2006): Ich-Konstruktionen in der Popliteratur - Christian Krachts „Faserland“ (1995), Alexa Hennig von Langes „Relax“ (1997) und Benjamin von Stuckrad-Barres „Soloalbum“ (1998). In: SCHLich, JuTTA / MEHRFORT, SANDRA (eds.): Individualität als Herausforderung. Heidelberg, 181-205.

PECHT, ANDREAS: Zoë Jenny kriegt die Kurve nicht: http://www.pecht.info/texte/2007/ 20071115.html (12.2012).

Plath, Sylvia (1990 / 1997): Die Glasglocke. Frankfurt (M.).

ZEH, Juli: Sag nicht Er zu mir oder: Vom Verschwinden des Erzählers im Autor. In: Akzente 08.2002:380f. 